\title{
A prospective study of CSF markers in 250 patients with possible Creutzfeldt-Jakob disease
}

\author{
B Van Everbroeck, S Quoilin, J Boons, J J Martin, P Cras
}

J Neurol Neurosurg Psychiatry 2003;74:1210-1214

See end of article for authors' affiliations .....................

Correspondence to: Dr Bart Van Everbroeck, Born Bunge Foundation, Laboratory of

Neurobiology, University of Antwerp, Universiteitsplein 1, B-2610 Wilriik,

Belgium;

bartve@uia.ua.ac.be

Received 30 September 2002

In revised form 11 April 2003

Accepted 12 April 2003

\begin{abstract}
Objective: To investigate various cerebrospinal fluid (CSF) markers that could assist in the clinical diagnosis of Creutzfeldt-Jakob disease (CJD).

Methods: CSF samples were analysed for the presence of 14-3-3 protein, microtubule associated protein tau, and $\beta$ amyloid in 250 patients with possible CJD. Densitometric analysis was used to quantify the level of 14-3-3 in all patients.

Results: Analysis of the clinical data showed that cerebellar signs or myoclonus combined with progressive dementia were the main features leading to a clinical suspicion of CJD. While 14-3-3 detection had a sensitivity of $100 \%$ and a specificity of $92 \%$, tau determination using a threshold of $1300 \mathrm{pg} / \mathrm{ml}$ had a sensitivity of $87 \%$ and a specificity of $97 \%$. If the protocol for the analysis of 14-3-3 was modified (using densitometric analysis) a higher specificity (97\%) could be obtained, but with a lower sensitivity (96\%). Maximum sensitivity, specificity, and positive predictive value were obtained with a combination of 14-3-3 and $\beta$ amyloid determinations. The concentrations of 14-3-3 and tau in the CSF were reduced in CJD patients with a long duration of disease (more than one year; $p<0.05$ ). The concentrations of 14-3-3 or tau were lowest at the onset or at the end stage of the disease, while the $\beta$ amyloid concentration remained low throughout the course of the disease.

Conclusions: Both 14-3-3 and tau protein are sensitive and specific biomarkers for CJD. The combination of 14-3-3 and $\beta$ amyloid analysis resulted in the maximum sensitivity, specificity, and positive predictive value. When these biomarkers are used in the diagnosis of CJD, the phase of the disease in which the CSF sample was obtained should be taken into account. Disease duration, dependent on the PrP genotype, also has a significant influence on the level of 14-3-3 and tau in the CSF.
\end{abstract}

$\mathrm{S}$ poradic Creutzfeldt-Jakob disease (CJD) is a rapidly progressive and ultimately fatal disorder belonging to the group of prion diseases. ${ }^{1}$ To date, a definitive diagnosis can only be made by postmortem neuropathological examination of brain tissue. ${ }^{2}$ Clinically, "possible" CJD is characterised by a rapidly progressive dementia (of less than two years' duration) together with at least two of the following symptoms: myoclonus, visual signs, extrapyramidal or pyramidal signs, akinetic mutism, and cerebellar signs. ${ }^{3}$ Patients who show a typical electroencephalogram (EEG) with triphasic repetitive slow wave complexes or a positive test for 14-3-3 protein are classified as "probable" CJD. ${ }^{4}$

Several proteins in the cerebrospinal fluid (CSF) can help in the clinical diagnosis of CJD. Immunodetection of the 14-3-3 protein $^{5}$ and tau protein ${ }^{6}$ has been reported to be the most sensitive and specific marker, but these proteins are also found in other diseases with overlapping clinical symptoms. Increases in neurone specific enolase ${ }^{7}$ or S-100 protein $^{8}$ have been reported, but are less sensitive and specific. ${ }^{9}$ Reduced concentrations of full length CSF $\beta$ amyloid 1-42 $\left(\mathrm{A} \beta_{1-42}\right)$ are often found in CJD patients, while they remain normal or may be increased in other neurological disorders with biochemical test results and clinical signs that mimick CJD. ${ }^{10}{ }^{11}$

After the description of a variant form of $\mathrm{CJD}^{12}$ and subsequent experiments establishing the link with bovine spongiform encephalopathy, ${ }^{13}{ }^{14}$ a surveillance network was formed in Belgium to monitor the incidence of CJD. As part of this surveillance network, our laboratory examined 250 CSF samples of clinically suspected CJD patients. For all these patients the referring physician provided a summary of the clinical record including the history, symptoms at onset, and follow up information.

In this study we evaluated the use of CSF biomarkers and analysed clinical and laboratory variables. First, we examined the clinical symptoms in both CJD and control groups to determine the criteria on which a patient was suspected of suffering from CJD. Second, we investigated which CSF markers, alone or in combination, were most useful. Third, we carried out a densitometric quantification of the 14-3-3 immunoblot analysis. Finally, we analysed possible influences such as age, disease duration, time of the lumbar puncture, and genetic polymorphism at codon 129 of the prion protein gene (PRNP) on CSF biomarkers. For example, it has been shown that the level of tau protein depends on the age of the patient. ${ }^{15}$ These results are important for quality control and also allow the development of a clinical diagnostic protocol.

\section{METHODS}

The prospective CSF study was initiated in September 1998 and all samples obtained until the 30 June 2002 were included in the present analysis. We received 250 CSF samples from patients who had clinical symptoms compatible with the diagnosis of "possible" CJD at the time of the lumbar puncture. The diagnosis of CJD was made according to standard criteria. ${ }^{16}$ The clinical diagnosis was neuropathologically confirmed by standard methods. ${ }^{17}$ If follow up information suggested an alternative diagnosis, the appropriate diagnostic criteria were used-for example, for Alzheimer's disease the criteria were those of the National Institute of Communicative Disorders and Stroke and the Alzheimer Disease and related Disorders Association (NINCDS-ADRDA). ${ }^{18}$

Immunoassay for 14-3-3 protein was done as described by Zerr et al, with minor modifications. ${ }^{19}{ }^{20}$ Briefly, $20 \mu \mathrm{l}$ of CSF were subjected to sodium dodecyl sulphate/polyacrylamide gel electrophoresis ( $\mathrm{pH} 8.2$ ) (6\% stacking/12\% separating gel at $75 \mathrm{~V}$ for 60 minutes) using a Mini-Protean ${ }^{\circledR}$ electrophoresis cell (Bio-Rad, Hercules, California, USA). The proteins were 
transferred to a nitrocellulose membrane (Hybond; Amersham, Biosciences Europe, Freiburg, Germany) at $15 \mathrm{~V}$ for 24 minutes using a Trans-blot ${ }^{\circledR}$ semi-dry electrophoretic transfer cell (Bio-Rad). A molecular weight marker set (Kaleidoscope prestained standards, Bio-Rad) and a standard positive sample (a brain homogenate) were used in all experiments. Immunostaining was done after blocking with phosphate buffered saline ( $\mathrm{pH} 7.5$ ) containing $2 \%$ non-fat milk, by incubation with anti-14-3-3 $\gamma$ polyclonal rabbit antibody (Santa Cruz Biotechnology sc-718, Santa Cruz, California, USA) at a 1:2000 dilution. Subsequently, the membrane was incubated with an alkaline phosphatase conjugated swine anti-rabbit IgG antibody (Dako D0306, Glostrup, Denmark) at a 1:2000 dilution. The antigen was detected by a colorimetric reaction using $0.02 \%$ nitroblue tetrazolium (Sigma, St Louis, Missouri, USA) and $0.02 \%$ X-phosphate/5-bromo-4-chloro-3-indolyl phosphate (Boehringer, Ingelheim, Germany). The blot was scored for the presence or absence of an immunoreactive band at $30 \mathrm{kDa}$. A band was identified as "strong" when it was equal to stronger than the band of the positive control. Other bands of lesser intensity were labelled "weak". For each signal the integrated optical density was measured and quantified using a Kodak image station 440 and accompanying software. These measurements were used to calculate the ratio of each signal to the positive standard, which was identical on all blots. The result of this calculation was expressed in arbitrary units (AU).

The $\mathrm{A} \beta_{1-42}$ concentration was determined as previously described..$^{121}$ The tau protein concentration was measured in duplicate by an enzyme linked immunosorbent assay (Innotest hTau-Ag, K-1032; Innogenetics, Ghent, Belgium). ${ }^{22}$ The PRNP codon 129 polymorphism was determined as previously described. ${ }^{23}$

\section{Data analysis}

In the analysis of influences on the CSF biomarkers and the clinical characteristics, we pooled the data on all three control groups (see below)-Alzheimer's disease, other dementias, and neurological disorders-into one control group. We only used the densitometric 14-3-3 data in the analysis. To study the influence of the time point of sampling in the course of the disease, we chose three arbitrary phases: "early", the first $25 \%$; "late", the final 15\%; and "middle", between the $25 \%$ and $85 \%$ of the total duration of the disease. These choices were made to enhance the clarity of the results and to simplify statistical analysis.

The Kolmogorov-Smirnov test (InStat software package) showed that the results obtained did not have a normal distribution $(p<0.05)$. Therefore analysis of continuous variables was done using centiles and the Mann-Whitney U test. For nominal variables Fisher's exact test was used. The relation between different variables and the levels of the biomarkers on a population basis was calculated using the Pearson test. Comparison of the results of the different markers was analysed using the $\chi^{2}$ test. Optimal cut off values were calculated using receiver operating characteristic (ROC) analysis.

\section{RESULTS}

\section{Clinical data}

Clinical data and CSF samples were available for 250 possible CJD patients. In 47 of these, the diagnosis of CJD was confirmed by neuropathology, while in five further probable cases of CJD this was impossible as permission for necropsy was refused. The mean (SD) age of the CJD group $(n=52)$ was $63(8)$ years.

In the remaining 198 patients other diagnoses were made using follow up information. The patients were assigned to three categories: Alzheimer's disease, other dementias, and other neurological disorders in which dementia is a secondary symptom. The first group consisted of 65 Alzheimer's disease patients, mean (SD) age 71 (8) years. Sixty two patients with other dementias formed the second group; these had the following diagnoses: vascular dementia $(n=26)$, frontal lobe dementia $(n=13)$, dementia with Lewy bodies $(n=23)$. The mean age of this group was 69 (8) years. The third group consisted of 71 patients with other neurological disorders and included viral encephalitis $(n=18)$, Hashimoto's encephalitis $(\mathrm{n}=6)$, multiple system atrophy $(\mathrm{n}=5)$, multiple sclerosis $(n=3)$, stroke $(n=10)$, metabolic dysfunction $(n=12)$, paraneoplastic syndrome $(\mathrm{n}=10)$, and psychiatric dysfunction $(\mathrm{n}=9)$. The average age of this group was 64 (13) years. There was no significant difference in age between these three groups.

\section{Biochemical determinations}

For all biomarker analysis we first examined the day to day variance for eight samples (four definite CJD, two Alzheimer's disease, one dementia with Lewy bodies, and one with multiple sclerosis) on three consecutive days and one week later. Visual analysis of 14-3-3 protein always resulted in the same evaluation. In the densitometric analysis of the blots, a variance of $3 \%$ was found. Tau and $A \beta_{1-42}$ concentrations were comparable to published results, with a variance less then $5 \% .^{10112224-26}$

The 14-3-3 protein was present in all CJD patients. A positive signal was also found in patients with vascular dementia and in five with viral encephalitis. A weak 14-3-3 band was observed in three patients with dementia induced by metabolic dysfunction, in three with dementia with Lewy bodies, and in two with Alzheimer's disease.

To standardise the interpretation of the immunoblots, we undertook densitometric analysis of the signals obtained. In the CJD patients a mean of $1.44 \mathrm{AU}$ with a range of 0.25 to 8.52 AU was found. In the control groups (pooled data), the mean was $0.00 \mathrm{AU}$ with a range of 0.00 to $0.86 \mathrm{AU}$. The signal density differed significantly between the CJD patients and the control groups $(\mathrm{p}<0.01)$. If we selected only the positive samples identified in the control groups, a mean of $0.48 \mathrm{AU}$ with a range from 0.12 to $0.86 \mathrm{AU}$ was found; this was significantly different from the CJD group $(\mathrm{p}=0.02)$. The maximum sensitivity (96\%) and specificity (97\%) were obtained when a cut off value of $0.60 \mathrm{AU}$ was used (ROC analysis).

\section{Value of CSF markers for clinical diagnosis}

A cut off value of $1300 \mathrm{pg} / \mathrm{ml}$ was used for analysis of the CSF tau protein concentration. ${ }^{26}$ We found an increased specificity (98\%) but a lesser sensitivity (87\%) compared with the 14-3-3 immunoblot. Separately, we investigated differences between the control groups and observed an increased concentration of tau protein in Alzheimer's disease $(625 \mathrm{pg} / \mathrm{ml})$ compared with other neurological disorders $(450 \mathrm{pg} / \mathrm{ml})(\mathrm{p}=0.05)$.

No significant difference in concentration of CSF A $\beta_{1-42}$ was observed between the CJD and control groups (304 and 293 $\mathrm{pg} / \mathrm{ml}$, respectively). We did identify a significantly increased concentration of $A \beta_{1-42}$ in control patients with a false positive $14-3-3$ result $(605 \mathrm{pg} / \mathrm{ml}, \mathrm{p}=0.04)$. Both the Alzheimer's disease group $(246 \mathrm{pg} / \mathrm{ml})$ and the other dementias group (289 $\mathrm{pg} / \mathrm{ml}$ ) had a significantly decreased level of $\mathrm{A} \beta_{1-42}$ compared with the neurological disease control group (346 pg/ml) $(\mathrm{p}<0.05)$.

To identify the best single test, we compared the 14-3-3 and tau detection rates (table 1 ). We found that densitometric analysis of 14-3-3 gave the best overall result but was not significantly different from the other 14-3-3 tests used. The 14-3-3 test had higher sensitivity than tau, whereas tau had a higher specificity $(\mathrm{p}<0.02$; table 1$)$.

If we combined the analysis of tau or 14-3-3 with $\mathrm{A} \beta_{1-42}$, using a cut off value of $400 \mathrm{pg} / \mathrm{ml}$, we obtained a significantly higher specificity ( $p=0.01$; table 1 ) and an unchanged sensitivity for 14-3-3, while the results obtained with the tau determination did not change. A combination of 14-3-3 and tau analysis did not enhance sensitivity or specificity. 
Table 1 Comparison of the biomarkers results using the number of true positive, true negative, false positive, and false negative patients; using these figures the sensitivity, specificity, positive predictive value, and negative predictive value were determined

\begin{tabular}{lllllllll}
\hline & TP & TN & FP & FN & Sens & Spec & PPV & NPV \\
\hline All 14-3-3 bands & 52 & 183 & 15 & 0 & $100 \%$ & $92 \%$ & $78 \%$ & $100 \%$ \\
Only strong bands & 49 & 191 & 7 & 3 & $94 \%$ & $96 \%$ & $88 \%$ & $98 \%$ \\
Densitometric analysis & 50 & 192 & 6 & 2 & $96 \%$ & $97 \%$ & $89 \%$ & $99 \%$ \\
$14-3-3$ and $A \beta_{1-42}{ }^{*}$ & 52 & 194 & 4 & 0 & $100 \%$ & $98 \%$ & $93 \%$ & $100 \%$ \\
Tau & 45 & 193 & 5 & 7 & $87 \%$ & $97 \%$ & $90 \%$ & $97 \%$ \\
Tau and $A \beta_{1.42}$ & 45 & 194 & 4 & 7 & $87 \%$ & $98 \%$ & $92 \%$ & $97 \%$ \\
\hline
\end{tabular}

*Best overall result.

FN, false negative; FP, false positive; NPV, negative predictive value; PPV, positive predictive value; sens, sensitivity; spec, specificity; TN, true negative; TP, true positive.

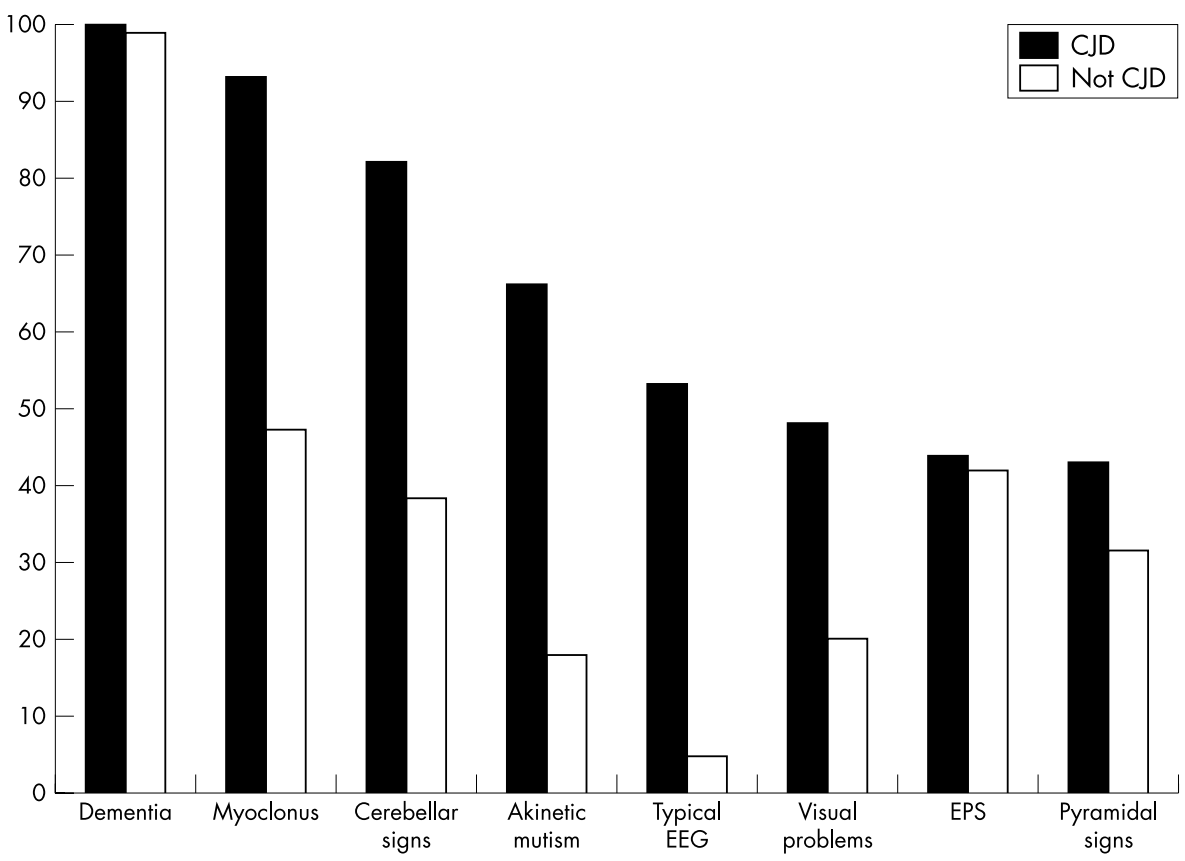

Figure 1 Prevalence of the signs and symptoms in definite Creutzfeldt-Jakob disease and control patients. EEG, electroencephalogram; EPS, extrapyramidal signs.

Although dementia was present in all patients, some initially had neurological or psychiatric signs. Myoclonus, visual problems, and cerebellar signs were most often identified (fig 1) and at least one of these was present in all patients. Visual problems were significantly more common in the CJD group $(p=0.03)$. An equal frequency of pyramidal and extrapyramidal signs was found in all groups. Typical EEG changes (sensitivity 50\%, specificity $82 \%$ ) and akinetic mutism, on the other hand, were rarely described in the control patients (fig 1).

We further compared the level of all biomarkers between samples that were sent cooled $\left(\right.$ at $\left.4^{\circ} \mathrm{C}\right)$ or frozen and those sent at room temperature (table 2). In the CJD group we analysed whether the time the samples were kept at room temperature during transport had any influence on the results, but no significant differences were observed.

Next we analysed the influence of age at the time of sampling (table 3). We did not observe significant differences for any marker. For the analysis of the disease duration, two different measurements were used. For CJD, which is invariably fatal, we used the total disease duration, whereas in the control groups we used the duration of the disease up to the point of sampling. This was done because some patients are still alive and thus the final duration is not yet known, and

Table 2 Comparison of the level of biomarkers between cooled transport (frozen or $4^{\circ} \mathrm{C}$ ) and transport at room temperature

\begin{tabular}{lllllll}
\hline & CJD group & \multicolumn{3}{c}{ Control group } & \\
\cline { 2 - 3 } Biomarker & Cooled $(n=20)$ & RT $(n=32)$ & & Cooled $(n=65)$ & RT $(n=133)$ & p Value \\
\hline $14-3-3$ positive & $20(100 \%)$ & $32(100 \%)$ & & $6(9 \%)$ & $9(7 \%)$ & $>0.7$ \\
Tau $(\mathrm{pg} / \mathrm{ml})$ & 12426 & 14250 & & 768 & 663 & $>0.2$ \\
A $\beta(\mathrm{pg} / \mathrm{ml})$ & 297 & 309 & 282 & 321 & $>0.4$ \\
\hline
\end{tabular}

A p value was calculated for both groups using Fisher's exact test for the 14-3-3 results and the Mann-Whitney $U$ test for tau (median) and $\beta$ amyloid (A $\beta$, median).

CJD, Creutzfeldt-Jakob disease; RT, room temperature. 
Table 3 Influence of age, disease duration, and prion protein gene PRNP 129 on the level of CSF biomarkers

\begin{tabular}{|c|c|c|c|c|c|c|c|c|}
\hline & \multicolumn{4}{|c|}{ CJD group } & \multicolumn{4}{|c|}{ Control group } \\
\hline & $\mathrm{n}$ & $\begin{array}{l}14-3-3 \\
(\mathrm{AU})\end{array}$ & $\begin{array}{l}\text { Tau } \\
\text { (pg/ml) }\end{array}$ & $\begin{array}{l}\mathrm{A} \beta \\
(\mathrm{pg} / \mathrm{ml})\end{array}$ & $\mathrm{n}$ & $\begin{array}{l}14-3-3 \\
\text { (AU) }\end{array}$ & $\begin{array}{l}\text { Tau } \\
\text { (pg/ml) }\end{array}$ & $\begin{array}{l}\mathrm{A} \beta \\
(\mathrm{pg} / \mathrm{ml})\end{array}$ \\
\hline \multicolumn{9}{|l|}{ Age } \\
\hline$<50$ years & 14 & 1.28 & 10250 & 224 & 50 & 0.02 & 412 & 369 \\
\hline $50-69$ years & 20 & 1.52 & 10500 & 343 & 55 & 0.04 & 584 & 242 \\
\hline$>69$ years & 13 & 1.48 & 12525 & 381 & 93 & 0.03 & 697 & 286 \\
\hline \multicolumn{9}{|l|}{ Disease duration } \\
\hline$<4$ months & 18 & $1.68^{*}$ & $11400 *$ & 274 & 35 & $0.04 *$ & $758^{*}$ & 355 \\
\hline 4-12 months & 20 & $1.99 *$ & $12650 *$ & 350 & 78 & $0.06^{*}$ & $594^{*}$ & 254 \\
\hline$>12$ months & 9 & $0.89 *$ & $2875^{*}$ & 341 & 95 & $0.00 *$ & $469 *$ & 235 \\
\hline PRNP 129 & & & & & \multicolumn{4}{|c|}{ Not done } \\
\hline Met/Met & 33 & $1.93 *$ & $14650 *$ & 302 & & & & \\
\hline Met/Val, $\mathrm{Val} / \mathrm{Val}$ & 19 & $0.96^{*}$ & $3250 *$ & 257 & & & & \\
\hline
\end{tabular}

Table 4 Influence of the time point of sampling in the disease course on the level of CSF biomarkers

\begin{tabular}{|c|c|c|c|c|c|c|c|c|}
\hline \multirow[b]{2}{*}{ Time point } & \multicolumn{4}{|c|}{ CJD group } & \multicolumn{4}{|c|}{ Control group } \\
\hline & $\mathrm{n}$ & $\begin{array}{l}14-3-3 \\
(A U)\end{array}$ & $\begin{array}{l}\text { Tau } \\
(p g / m l)\end{array}$ & $\underset{(\mathrm{pg} / \mathrm{ml})}{\mathrm{A} \beta}$ & $\mathrm{n}$ & $\begin{array}{l}14-3-3 \\
(\mathrm{AU})\end{array}$ & $\begin{array}{l}\text { Tau } \\
(\mathrm{pg} / \mathrm{ml})\end{array}$ & $\begin{array}{l}\mathrm{A} \beta \\
(\mathrm{pg} / \mathrm{ml})\end{array}$ \\
\hline Early & 6 & $0.80^{*}$ & $2750 *$ & 230 & 12 & 0.00 & 312 & 298 \\
\hline Middle & 39 & 2.01 * & 14850 * & 316 & 50 & 0.05 & 928 & 345 \\
\hline Late & 7 & $0.89 *$ & 2200 * & 257 & 13 & 0.00 & 352 & 201 \\
\hline
\end{tabular}

also because some patients recovered. We found significantly decreased levels of tau and 14-3-3 proteins in patients with a long disease duration in both groups $(\mathrm{p}<0.03)$ (table 3$)$. When these calculations were done for the different control groups separately, the decrease levels could still be observed but to a lesser degree because of the smaller sample sizes $(\mathrm{p}=0.05$ for tau and $\mathrm{p}=0.06$ for $14-3-3)$.

We also examined the influence of PRNP codon 129 polymorphism on the levels of CSF biomarkers in CJD patients. We found reduced levels of 14-3-3 and tau proteins in patients with Met/Val $(\mathrm{n}=12)$ and Val/Val $(\mathrm{n}=7)$ compared with Met/Met $(n=33)$. Owing to the small sample size this difference was only found to be significant when the data on Met/Val and Val/Val patients were pooled (table 3 ). When multivariate analysis was done to correct for the influence of disease duration this effect was no longer significant.

Finally, we assessed the influence of the time point of sampling in the course of the disease. We examined pooled data on CJD $(n=42)$ and control patients $(n=75)$ in whom total disease duration and the phase in which the sample was taken were known. We found that for CJD patients, samples taken early and late in the course of the disease had significantly decreased levels of 14-3-3 and tau compared with samples taken in the middle part of the disease duration $(\mathrm{p}<0.001$; table 4). For control patients this effect was only seen for tau protein $(\mathrm{p}<0.02$; table 4$)$. When multivariate analysis was done to correct for the influence of the total duration of the disease this effect was still significant $(p<0.05)$.

In five CJD patients we received two successive samples. In this small group a significant difference could also be found in the 14-3-3 and tau levels ("early": 14-3-3 protein, 0.79 AU; tau protein, $1290 \mathrm{pg} / \mathrm{ml}$; "middle": $14-3-3$ protein, $1.52 \mathrm{AU}$; tau protein, $8923 \mathrm{pg} / \mathrm{ml} ; \mathrm{p}<0.04)$.

\section{DISCUSSION}

In this paper we report the results of a prospective study on CSF undertaken in our laboratory in collaboration with the
Belgian CJD surveillance network. We studied the 14-3-3 test and other markers in CSF as tools for the differential diagnosis. We examined the results of the 14-3-3 immunoblot both by visual analysis and densitometric analysis and found no significant differences.

Analysis of the symptoms at the time the sample was delivered showed that the combination of dementia with cerebellar signs or myoclonus most often raised suspicion of CJD. The most specific clinical symptoms for the differential diagnosis were akinetic mutism and periodic sharp wave complexes on the EEG. Follow up information showed that Alzheimer's disease was the most common differential diagnosis, followed by vascular dementia.

The 14-3-3 test ${ }^{51927}$ and the tau protein determination $^{62425}$ (cut off $1300 \mathrm{pg} / \mathrm{ml}$ ) are both reliable assays for use in the clinical diagnosis of CJD. In this series, we observed that the 14-3-3 test was more sensitive (100\%) than the tau determination (87\%). On the other hand, the tau determination had a greater specificity (98\%) than the 14-3-3 test (93\%). These results are in accordance with those obtained in similar studies. The reported concentrations of $\mathrm{A} \beta_{1-42}$ and tau in our control group were different from those found in other published reports, ${ }^{96}$ but when we analysed $\mathrm{A} \beta_{1-42}$ or tau for each control group separately, we found comparable results. We confirmed the reported early rise of tau in CJD patients, but also identified low levels of 14-3-3 protein.

Our study confirms that the concentration of CSF $A \beta_{1-42}$ is increased in some neurological conditions that can have symptoms suggestive of CJD and positive 14-3-3 or tau tests. $^{10}{ }^{11}$ The 14-3-3 test combined with the detection of $\mathrm{A} \beta_{1-42}$ resulted in a significantly increased specificity $(98 \%$, $\mathrm{p}=0.01)$. This is important in differentiating treatable diseases from clinically probable CJD.

We found no influence on the results of the samples being kept for prolonged periods at room temperature, or of the way the samples were send to the laboratory, or of the age of the 
patient. On the other hand, disease duration and PRNP codon 129 had a significant but related influence on the results of both 14-3-3 and tau protein analyses. This is because most patients with a long duration of disease (more than one year) also had the Met/Val or Val/Val genotype. We concluded that samples taken early or late in the course of the disease could produce false negative or weak positive results.

We would therefore like to propose the following strategy in relation to possible or probable CJD in patients with negative biomarker analysis. If the clinical signs are still suggestive of CJD after three months, repeat analysis should be considered. At the same time it might also be useful to analyse the PRNP codon 129 as this influences both the biomarker analyses and the disease duration.

\section{Conclusions}

Detection of CSF tau protein had the highest positive predictive value, whereas detection of 14-3-3 protein had the best negative predictive value. Both test are equally valuable as tools in CJD diagnosis (table 1). Our study further showed that the level of $14-3-3$ and tau proteins in the CSF was significantly reduced in CJD patients with a long disease duration (more than one year). Independently of this finding we also observed that the levels of 14-3-3 or tau were highest in the "middle" phase of the disease course, while decreased concentrations were found in the "early" and "late" phases, sometimes generating false negative results. These variations were not observed with the $A \beta_{1-42}$ values.

\section{ACKNOWLEDGEMENTS}

We thank all physicians who sent us the CSF samples and clinical information on suspected CJD patients and control cases, and the Fund for Scientific Research (FWO) for supporting the project. We further thank Prof Dr C Van Broeckhoven for technical assistance with the genotyping and densitometric analysis. BVE is a postdoctoral researcher of the Fund for Scientific Research (FWO).

\section{Authors' affiliations}

B Van Everbroeck, J Boons, J J Martin, P Cras, Born Bunge

Foundation, University of Antwerp, Wilrijk, Belgium

S Quoilin, Institute of Public Health Louis Pasteur, Brussels, Belgium

Competing interests: none declared

\section{REFERENCES}

1 Prusiner SB, Hsiao KK. Human prion diseases. Ann Neurol 1994; 35:385-95.

2 Budka H, Aguzzi A, Brown P, et al. Neuropathological diagnostic criteria for Creutzfeldt-Jakob disease (CJD) and other human spongiform encephalopathies (prion diseases). Brain Pathol 1995;5:459-66.

3 Masters CL, Harris JO, Gajdusek DC, et al. Creutzfeldt-Jakob disease: patterns of worldwide occurrence and the significance of familial and sporadic clustering. Ann Neurol 1979;5:177-88.

4 Poser S, Mollenhaver B, Krauss A, et al. How to improve the clinical diagnosis of Creutzfeldt-Jakob disease. Brain 1999;12:2345-51.

5 Hsich G, Kenney K, Gibbs CJ, et al. The 14-3-3 brain protein in cerebrospinal fluid as a marker for transmissible spongiform encephalopathies. N Engl J Med 1996;335:924-30.
6 Otto $M$, Wiltfang J, Tumani $\mathrm{H}$, et al. Elevated levels of tau-protein in cerebrospinal fluid of patients with Creutzfeldt-Jakob disease. Neurosci Lett 1997;225:210-12

7 Zerr I, Bodemer M, Racker S, et al. Cerebrospinal fluid concentration of neuron-specific enolase in diagnosis of Creutzfeldt-Jakob disease. Lancet 1995;345: 1609-10.

8 Otto $M$, Stein H, Szudra A, et al. S-100 protein concentration in the cerebrospinal fluid of patients with Creutzfeldt-Jakob disease. J Neurol 1997;244:566-70.

9 Beaudry P, Cohen P, Brandel JP, et al. 14-3-3 protein, neuron-specific enolase, and S-100 protein in cerebrospinal fluid of patients with Creutzfeldt-Jakob disease. Dement Geriatr Cogn Disord 1999;10:40-6.

10 Van Everbroeck B, Green A, Pals P, et al. Decreased levels of amyloid-beta in cerebrospinal fluid of Creutzfeldt-Jakob patients. J Alzheimer Dis 1999;1:343-7.

11 Otto M, Esselmann H, Schulz-Shaeffer W, et al. Decreased beta-amyloid 1-42 in cerebrospinal fluid of patients with Creutzfeldt-Jakob disease. Neurology 2000;54:1099-102.

12 Will RG, Ironside JW, Zeidler $M$, et al. A new variant of Creutzfeldt-Jakob disease in the UK. Lancet 1996;347:921-5.

13 Collinge J, Sidle KC, Meads J, et al. Molecular analysis of prion strain variation and the aetiology of "new variant" CJD. Nature 1996:383:685-90.

14 Bruce ME, Will RG, Ironside JW, et al. Transmissions to mice indicate that "new variant" CJD is caused by the BSE agent. Nature 1997;389:498-501.

15 Vanmechelen E, Vanderstichele H, Hulstaert F, et al. Cerebrospinal fluid tau and beta-amyloid ((1-42)) in dementia disorders. Mech Ageing Dev $2001 ; 122: 2005-11$.

16 Weber T. Clinical and laboratory diagnosis of Creutzfeldt-Jakob disease. Clin Neuropathol 2000;19:249-50.

17 Van Everbroeck B, Pals P, Dziedzic T, et al. Retrospective study of Creutzfeldt-Jakob disease in Belgium: neuropathological findings. Acta Neuropathol 2000;99:358-64.

18 McKhann G, Drachman D, Folstein M, et al. Clinical diagnosis of Alzheimer's disease: report of the NINCDS-ADRDA work group under the auspices of Department of Health and Human Services task force on Alzheimer's disease. Neurology 1984;34:939-44.

19 Zerr I, Bodemer M, Weber T. The 14-3-3 brain protein and transmissible spongiform encephalopathy. N Engl J Med 1997;336:874; discussion, $874-5$

20 Zerr I, Bodemer M, Gefeller O, et al. Detection of 14-3-3 protein in the cerebrospinal fluid supports the diagnosis of Creutzfeldt-Jakob disease. Ann Neurol 1998;43:32-40.

21 Vanderstichele H, Blennow K, D'Heuvaert N, et al. Development of a specific diagnostic test for measurement of $\beta$-amyloid (1-42) in CSF. In: Fisher A, Hanin I, Yoshida M, eds. Progress in Alzheimer's and Parkinson's disease. New York: Plenum Press, 1998:773-8.

22 Blennow K, Wallin A, Agren $\mathrm{H}$, et al. Tau protein in cerebrospinal fluid: a biochemical marker for axonal degeneration in Alzheimer disease? Mol Chem Neuropathol 1995;26:231-45.

23 Van Everbroeck B, Croes EA, Pals P, et al. Influence of the prion protein and the apolipoprotein $E$ genotype on the Creutzfeldt-Jakob disease phenotype. Neurosci Lett 2001;313:69-72.

24 Green AJE, Thompson EJ, Stewart GE, et al. Use of 14-3-3 and other brain-specific proteins in CSF in the diagnosis of variant Creutzfeldt-Jakob disease. J Neurol Neurosurg Psychiatry $2001 ; 70: 744-8$.

25 Kapaki E, Kilidireas K, Paraskevas GP, et al. Highly increased CSF tau protein and decreased beta-amyloid (1-42) in sporadic CJD: a discrimination from Alzheimer's disease? J Neurol Neurosurg Psychiatry 2001;71:401-3.

26 Otto M, Wiltfang J, Cepek L, et al. Tau protein and 14-3-3 protein in the differential diagnosis of Creutzfeldt-Jakob disease. Neurology 2002;58: 192-7.

27 Brandel JP, Peoc'h K, Beaudry P, et al. 14-3-3 protein cerebrospinal fluid detection in human growth hormone-treated Creutzfeldt-Jakob disease patients. Ann Neurol 2001;49:257-60. 